\title{
Synthesis and Antimicrobial Activity of Some $[1,2,4]$-Triazole Derivatives
}

\author{
Bhimagouda S. Patil, ${ }^{1,2}$ G. Krishnamurthy, ${ }^{2}$ N. D. Shashikumar, \\ M. R. Lokesh, ${ }^{2}$ and H. S. Bhojya Naik ${ }^{3}$ \\ ${ }^{1}$ Syngene International Ltd., Biocon Park, Plot No. 2 \& 3, Bommasandra-Jigani Road, Karnataka, Bangalore 560100, India \\ ${ }^{2}$ Department of Chemistry, Sahyadri Science College, Karnataka, Shimoga 577451, India \\ ${ }^{3}$ Department of Industrial Chemistry, Kuvempu University, Karnataka, Shimoga 577451, India
}

Correspondence should be addressed to G. Krishnamurthy; gkmnaik_sahyadri@yahoo.co.in

Received 26 June 2012; Revised 17 September 2012; Accepted 24 September 2012

Academic Editor: George Psomas

Copyright (C) 2013 Bhimagouda S. Patil et al. This is an open access article distributed under the Creative Commons Attribution License, which permits unrestricted use, distribution, and reproduction in any medium, provided the original work is properly cited.

A series of novel [1,2,4]-triazolo piperidine (8), [1,2,4]-triazolo piperazine (9a-c), [1,2,4]-triazolo phenylether $(\mathbf{1 0 a}-\mathbf{e})$, and [1,2,4]triazolo aniline (11a-c) derivatives have been synthesized. The chemical structures of the newly synthesized compounds were characterized by IR, ${ }^{1} \mathrm{H}$ NMR, ${ }^{13} \mathrm{C}$ NMR, and LCMS. The newly synthesized compounds were screened for antimicrobial activity. Among all the compounds tested, $\mathbf{1 1} \mathbf{b}\left(\mathrm{R}_{4}=4-\mathrm{MeO}-\right)$ showed the highest activity against Staphylococcus aureus and Escherichia coli, and $9 \mathrm{a}\left(\mathrm{R}_{1}\right.$ and $\left.\mathrm{R}_{2}=\mathrm{Cl}\right)$ showed the highest activity against Pseudomonas aeruginosa.

\section{Introduction}

The antimicrobial agents available now have various drawbacks such as toxicity, drug resistance to microbes, and narrow spectrum of activity. Hence the design of new compounds to deal with the above problems has become one of the most challenging targets in antibacterial and antifungal research today.

1,2,4-Triazole derivatives are known to exhibit a wide range of biological activities, such as antibacterial [1-4] and antifungal [1-5], analgesic [6], anticancer [7, 8], antiviral [9], antitubercular $[10,11]$, anti-inflammatory [12], anticonvulsant [13, 14], antidepressant [15], and central nervous system (CNS) [16]. A number of 1,2,4-triazole-3-one and its heterocyclic derivatives represent an interesting class of compounds possessing a broad spectrum of biological activities like antimicrobial $[17,18]$, anticonvulsant $[19,20]$, and 5-HT2 antagonists [21]. Furthermore, 1,2,4-triazoles bearing piperazine substituents show biological activities, such as antibacterial [22, 23], antifungal [5, 22, 23], and anticancer [7]. Also, some halo phenylethers incorporating the 1,2,4-triazole nucleus possess good anticancer [24] and analgesic [25] activities.
1,2,4-Triazole and 1,2,4-triazol-3-one bearing piperazine substituent find application in the treatment of several diseases (Figure 1). Itraconazole and posaconazole are important antifungal drugs [26]. Also several compounds containing the piperazine nucleus are well known as drugs. For example Prazosin, Lidoflazine, and Urapidil are used as cardiovascular agents [27-29], in addition to Norfloxacin, Ciprofloxacin, and Ofloxacin, which are effective drugs against respiratory, urinary, gastrointestinal tracts, skin, and soft tissue infections caused by either Gram-positive or Gram-negative bacteria [30, 31].

In view of all these facts, the aim of the present study is to synthesize 1,2,4-triazole-3-one containing piperidine, piperazine, phenylether, and aniline with the hope that new antimicrobial agents will be developed.

1.1. Chemistry. The synthetic route for key intermediate methanesulfonic acid 2-[1-(4-methoxy-phenyl])-3-methyl5-oxo-1,5-dihydro-[1,2,4]-triazol-4-yl]-ethylester (7) was depicted in Scheme 1. This intermediate was prepared from 4-methoxy aniline through the sydnone by two ring transformation reaction following the literature method [32]. 

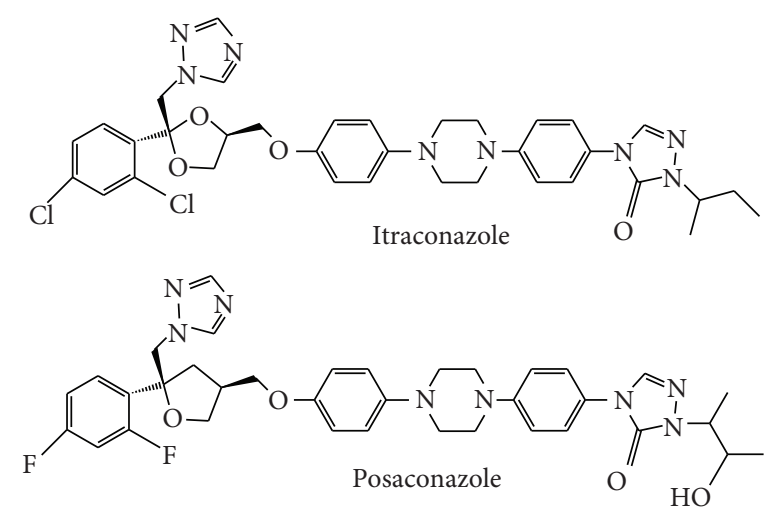

Figure 1: Drugs containing 1,2,4-triazole, 1,2,4-triazole-3-one, piperazine, and 2,4-dihalo phenyl moieties.

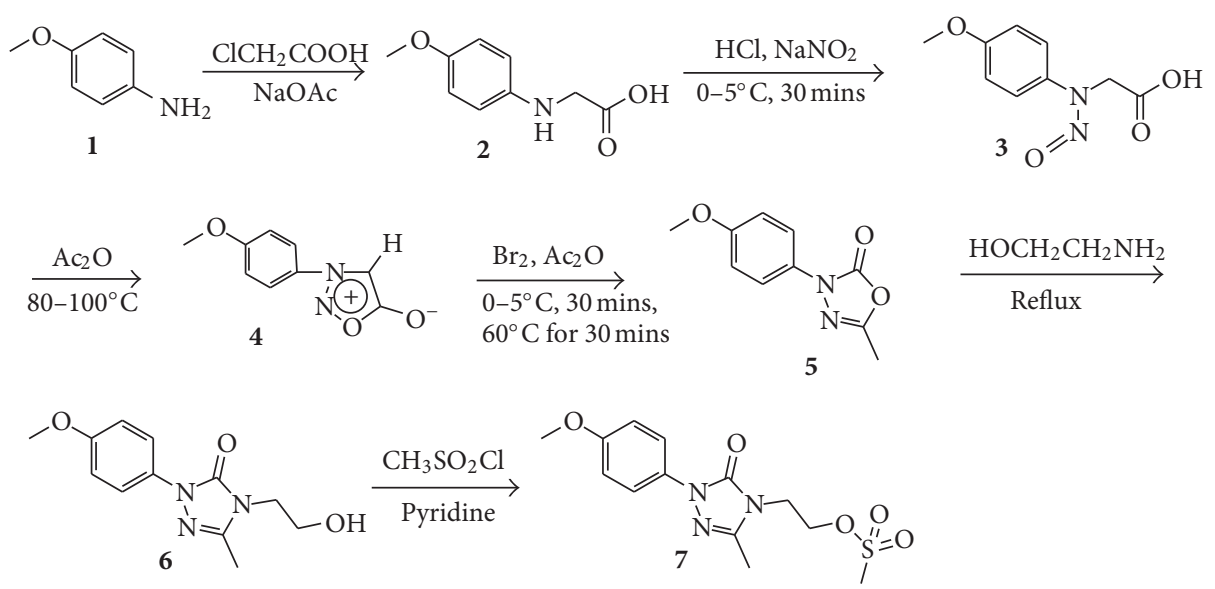

SCHEME 1: Synthetic route for the key intermediate.

The synthetic route followed for the preparation of the new compounds is outlined in Scheme 2. The reaction of methanesulfonic acid 2-[1-(4-methoxy-phenyl])-3-methyl5-oxo-1,5-dihydro-[1,2,4]-triazol-4-yl]-ethylester (7) with substituted piperidine, piperazines, and phenols in the presence of $\mathrm{Cs}_{2} \mathrm{CO}_{3}$ gave $[1,2,4]$-triazolo piperidine $(8),[1,2,4]$ triazolo piperazines $(\mathbf{9 a}-\mathbf{c})$ and $[1,2,4]$-triazolo phenylethers (10a-e) in moderate to good yields. We have tried similar conditions for anilines and instead of the desired products 11a-c, we obtained alkene (12), the structure of which was confirmed by ${ }^{1} \mathrm{H}$ NMR and LCMS. This is possible due to the fact that anilines are less reactive towards nucleophilic substitution. Hence in the presence of $\mathrm{Cs}_{2} \mathrm{CO}_{3}$ dehydromethylsulphonation is prefered over substitution reaction. Triethylamine first reacts with 7 and forms more reactive quaternary salt, which intern reacts with less nucleophilic anilines. We have obtained the desired products $11 \mathbf{a}-\mathbf{c}$ after changing the base from $\mathrm{Cs}_{2} \mathrm{CO}_{3}$ to triethyl amine.

\section{Experimental Section}

Melting points were determined on a Buchi B-545 melting point apparatus and are uncorrected. ${ }^{1} \mathrm{H}$ NMR spectra of the samples in $\mathrm{CDCl}_{3}$ and DMSO- $d_{6}$ were recorded on a Bruker Avance (300 or $400 \mathrm{MHz}$ ) spectrometer using tetramethylsilane (TMS) as an internal standard. ${ }^{13} \mathrm{C}$ NMR spectra were recorded in DMSO- $d_{6}$ at $100 \mathrm{MHz}$ with TMS as an internal reference. IR spectra were recorded on a Nicolet 6700 FT-IR spectrometry using ATR-technique (ATR = Attenuated Total Reflectance). C, H, N, and S analyses were carried out on an Elementor (Vario microcube). Mass spectra were recorded on a LC-MS-Agilent 1200 spectrometer.

2.1. General Procedure for the Synthesis of Piperidine (8) and Piperazine (9a-c) Derivatives. To a solution of compound (7) $(4 \mathrm{mmol})$ in DMF $(8 \mathrm{~mL})$ was added substituted piperidine/piperazines $(4.4 \mathrm{mmol}), \mathrm{Cs}_{2} \mathrm{CO}_{3}(6 \mathrm{mmol})$, and 


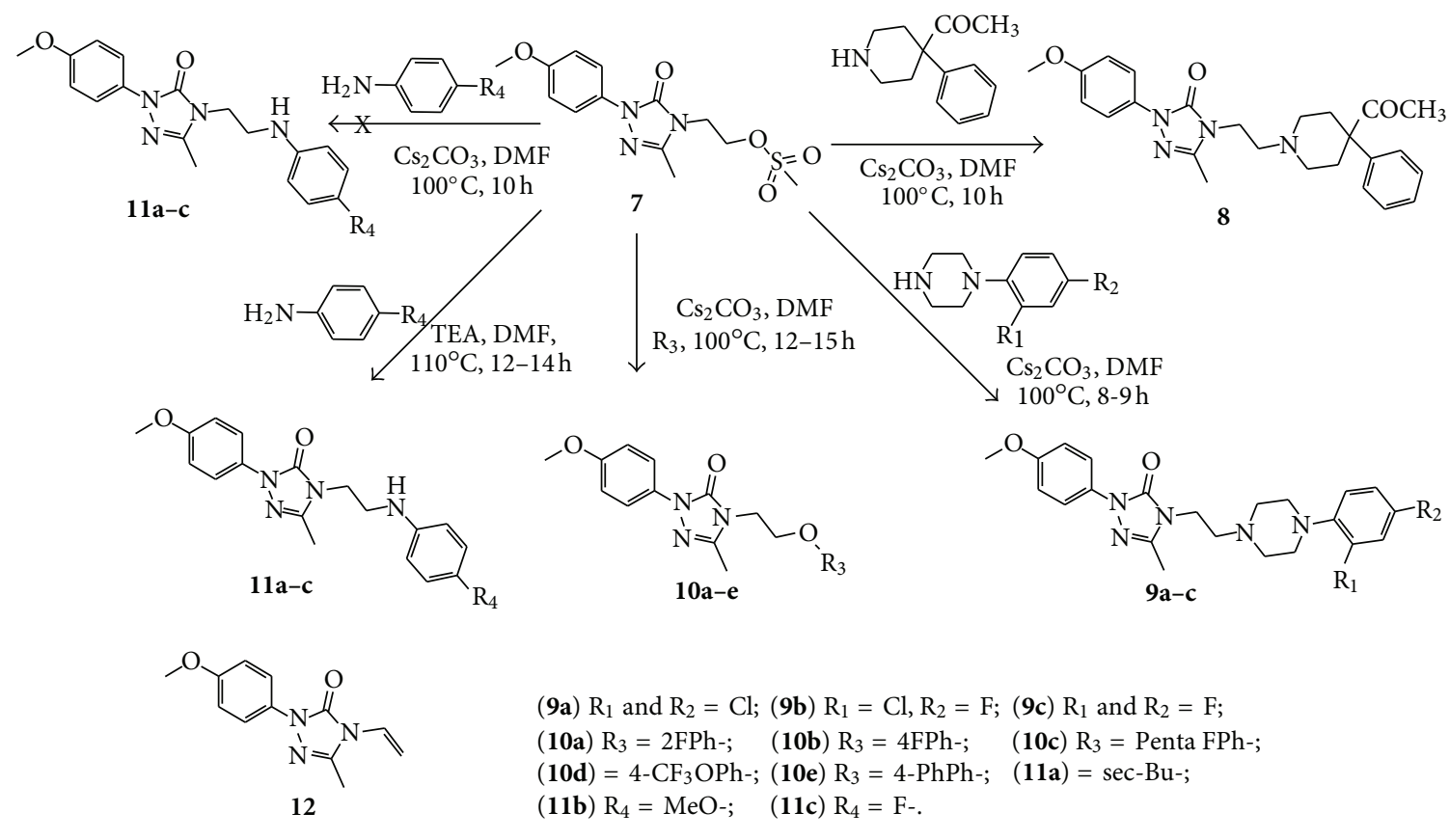

Scheme 2: Synthesis of [1,2,4]-triazolo piperidine, piperazine, phenylether, and aniline derivatives.

the mixture was stirred at $100^{\circ} \mathrm{C}$ for $14-15 \mathrm{~h}$. The progress of the reaction was monitored by TLC. After completion of the reaction, the mixture was poured on to ice water. The product was extracted with ethyl acetate $(2 \times 50 \mathrm{~mL})$. The combined organic phases were washed with water $(2 \times 40 \mathrm{~mL})$ and brine $(40 \mathrm{~mL})$. The organic phase was dried over sodium sulfate and the solvent was evaporated under reduced pressure to afford [1,2,4]-triazolo piperidine (8) and [1,2,4]-triazolo piperazine derivatives $(\mathbf{9 a}-\mathbf{c})$ in moderate to good yields.

4-[2-(4-Acetyl-4-phenylpiperidin-1-yl)-ethyl]-2-(4-methoxyphenyl)-5-methyl-2,4-dihydro[1,2,4]triazol-3-one (8).

Purified by column chromatography (Petroleum ether/ Ethyl acetate, 50/50); white solid, yield: $70 \%$, m.p. $102-106^{\circ} \mathrm{C}$. ${ }^{1} \mathrm{H}$ NMR $\left(400 \mathrm{MHz}, \mathrm{DMSO}-d_{6}\right): \delta 7.74(\mathrm{~d}, 2 \mathrm{H}, J=9.14 \mathrm{~Hz})$, 7.38-7.31 (m, 4H, Ar-H), 7.28-7.24 (m, 1H, Ar-H), 6.99 $(\mathrm{d}, 2 \mathrm{H}, J=9.14 \mathrm{~Hz}, \mathrm{Ar}-\mathrm{H}), 3.75\left(\mathrm{~s}, 3 \mathrm{H}, \mathrm{O}-\mathrm{CH}_{3}\right), 3.72-3.69$ $\left(\mathrm{m}, 2 \mathrm{H}\right.$, triazole- $\left.\mathrm{N}-\mathrm{CH}_{2}-\right), 2.68-2.62\left(\mathrm{~m}, 2 \mathrm{H}, \mathrm{N}-\mathrm{CH}_{2}-\right)$, 2.50-2.49 (m, $\left.2 \mathrm{H}, \mathrm{N}-\mathrm{CH}_{2}-\right), 2.38-2.34\left(\mathrm{~m}, 2 \mathrm{H}, \mathrm{N}-\mathrm{CH}_{2}-\right)$, $2.28\left(\mathrm{~s}, 3 \mathrm{H}, \mathrm{Ar}-\mathrm{CH}_{3}\right), 2.26-2.21\left(\mathrm{~m}, 2 \mathrm{H}, \mathrm{C}-\mathrm{CH}_{2}-\right), 1.93-1.91$ $\left(\mathrm{m}, 2 \mathrm{H}, \mathrm{C}-\mathrm{CH}_{2}-\right), 1.86\left(\mathrm{~s}, 3 \mathrm{H}, \mathrm{CO}-\mathrm{CH}_{3}\right) ;{ }^{13} \mathrm{C} \mathrm{NMR}$ $\left(100 \mathrm{MHz}, \mathrm{DMSO}-d_{6}\right): \delta 210.0,156.8,151.9,145.5,141.8$, $131.7,129.3,127.5,126.9,120,114.7,56.5,55.7,54.4,51.0$, 39.1, 32.7, 25.8, 11.9. MS: m/z 435 [m+1]; Anal. Calculated for $\mathrm{C}_{25} \mathrm{H}_{30} \mathrm{~N}_{4} \mathrm{O}_{3}$ : C. 69.10, H. 6.96, N. 12.89. Found C. 68.93, H. 6.97 , N. $12.92 \%$.

4-\{2-[4-(2,4-Dichlorophenyl)piperazin-1-yl]ethyl $\}-2-(4-$ methoxyphenyl)-5-methyl-2,4-dihydro[1,2,4]triazol-3-one (9a).

Purified by column chromatography (Petroleum ether/ Ethyl acetate, 50/50); off-white solid, yield: 60\%, m.p. $108-109^{\circ} \mathrm{C} .{ }^{1} \mathrm{H}$ NMR $\left(400 \mathrm{MHz}, \mathrm{DMSO}-d_{6}\right): \delta 7.75$ (d, $2 \mathrm{H}$,
$J=9.13 \mathrm{~Hz}, \mathrm{Ar}-\mathrm{H}), 7.53(\mathrm{~s}, 1 \mathrm{H}, \mathrm{Ar}-\mathrm{H}), 7.35(\mathrm{~d}, 1 \mathrm{H}, J=$ $8.6 \mathrm{~Hz}, \mathrm{Ar}-\mathrm{H}), 6.99$ (d, $2 \mathrm{H}, J=9.13 \mathrm{~Hz}, \mathrm{Ar}-\mathrm{H}), 3.79-3.75$ $\left(\mathrm{m}, 2 \mathrm{H}\right.$, triazole- $\left.\mathrm{N}-\mathrm{CH}_{2}-\right), 3.75\left(\mathrm{~s}, 3 \mathrm{H}, \mathrm{O}-\mathrm{CH}_{3}\right), 2.98-2.94$ (m, 4H, N-CH $\left.{ }_{2}-\right), 2.63-2.60\left(\mathrm{~m}, 6 \mathrm{H}, \mathrm{N}-\mathrm{CH}_{2}-\right), 2.33$ (s, $3 \mathrm{H}$, triazole- $\mathrm{CH}_{3}$ ). MS: $\mathrm{m} / \mathrm{z} 462[\mathrm{~m}+1]$; Anal. Calculated for $\mathrm{C}_{22} \mathrm{H}_{25} \mathrm{Cl}_{2} \mathrm{~N}_{5} \mathrm{O}_{2}$ : C. 57.15, H. 5.45, N. 15.15. Found C. 57.01, H. 5.47, N. 15.13 .

4-\{2-[4-(2-Chloro-4-fluorophenyl)piperazin-1-yl]ethyl\}2-(4-methoxyphenyl)-5-methyl-2,4-dihydro[1,2, 4] triazol3-one (9b).

Purified by column chromatography (Petroleum ether/ Ethyl acetate, 50/50); off-white solid, yield: 59\%, m.p. $115.8-117^{\circ} \mathrm{C} .{ }^{1} \mathrm{H}$ NMR $\left(400 \mathrm{MHz}, \mathrm{DMSO}-d_{6}\right): \delta 7.75(\mathrm{~d}, 2 \mathrm{H}$, $J=8.80 \mathrm{~Hz}, \mathrm{Ar}-\mathrm{H}), 7.20(\mathrm{t}, 1 \mathrm{H}, J=9.08 \mathrm{~Hz}, \mathrm{Ar}-\mathrm{H}), 7.08-7.06$ (m, 1H, A-H), 6.99 (d, 2H, J = 8.80 Hz, Ar-H), 6.95-6.91 $(\mathrm{m}, 1 \mathrm{H}, \mathrm{Ar}-\mathrm{H}), 3.80-3.74\left(\mathrm{~m}, 2 \mathrm{H}\right.$, triazole- $\left.\mathrm{N}-\mathrm{CH}_{2}-\right), 3.76(\mathrm{~s}$, $\left.3 \mathrm{H}, \mathrm{O}-\mathrm{CH}_{3}\right), 3.12-3.00\left(\mathrm{~m}, 4 \mathrm{H}, \mathrm{N}-\mathrm{CH}_{2}-\right), 2.57-2.59(\mathrm{~m}, 6 \mathrm{H}$, $\mathrm{N}-\mathrm{CH}_{2}-$ ), 2.33 (s, $3 \mathrm{H}$, triazole- $\mathrm{CH}_{3}$ ). MS: $\mathrm{m} / \mathrm{z} 446.2[\mathrm{~m}+1]$; Anal. Calculated for $\mathrm{C}_{22} \mathrm{H}_{25} \mathrm{ClFN}_{5} \mathrm{O}_{2}$ : C. 59.26, H. 5.65, N. 15.71. Found C. 59.40, H. 5.62, N. 15.70\%.

4-\{2-[4-(2,4-Difluorophenyl)piperazin-1-yl]ethyl\}-2-(4methoxyphenyl)-5-methyl-2,4-dihydro[1,2,4]triazol-3-one (9c).

Purified by column chromatography (Petroleum ether/ Ethyl acetate, 50/50); off-white solid; yield: 62\%, m.p. 101-102.4 ${ }^{\circ} \mathrm{C} .{ }^{1} \mathrm{H}$ NMR $\left(400 \mathrm{MHz}, \mathrm{DMSO}-d_{6}\right): \delta 7.75$ (d, $2 \mathrm{H}, J=9.13 \mathrm{~Hz}), 7.20-7.14(\mathrm{~m}, 1 \mathrm{H}, \mathrm{Ar}-\mathrm{H}), 7.07-7.03(\mathrm{~m}$, $1 \mathrm{H}, \mathrm{Ar}-\mathrm{H}), 6.99(\mathrm{~d}, 2 \mathrm{H}, J=9.14 \mathrm{~Hz}, \mathrm{Ar}-\mathrm{H}), 6.98-6.95(\mathrm{~m}$, $1 \mathrm{H}, \mathrm{Ar}-\mathrm{H}), 3.77-3.74\left(\mathrm{~m}, 2 \mathrm{H}\right.$, triazole- $\left.\mathrm{N}-\mathrm{CH}_{2}-\right), 3.75(\mathrm{~s}$, $\left.3 \mathrm{H}, \mathrm{O}-\mathrm{CH}_{2}-\right), 2.93\left(\mathrm{~m}, 4 \mathrm{H}, \mathrm{N}-\mathrm{CH}_{2}-\right), 2.61-2.57(\mathrm{~m}, 6 \mathrm{H}$, $\left.\mathrm{N}-\mathrm{CH}_{2}-\right), 2.32\left(\mathrm{~s}, 3 \mathrm{H}, \mathrm{N}-\mathrm{CH}_{2}-\right) ;{ }^{13} \mathrm{C}$ NMR $(100 \mathrm{MHz}$, 
DMSO- $\left.d_{6}\right): \delta 157.3,156.8,155.1,151.9,137.1,131.6,120.5$, $119.9,114.6,111.4,115.1,56.3,55.7,53.3,50.7,38.6,12.0$. MS: $\mathrm{m} / \mathrm{z} 430.2[\mathrm{~m}+1]$; Anal. Calculated for $\mathrm{C}_{22} \mathrm{H}_{25} \mathrm{~F}_{2} \mathrm{~N}_{5} \mathrm{O}_{2}$ : C. 61.53 , H. 5.87, N. 16.31. Found C. 61.48, H. 5.89, N. $16.29 \%$.

2.2. General Procedure for the Synthesis of Phenol Derivatives (10a-e). A mixture of compound (7) (4 mmol), the appropriate substituted phenols $(4.4 \mathrm{mmol}), \mathrm{Cs}_{2} \mathrm{CO}_{3}(6 \mathrm{mmol})$, and $\mathrm{CuI}(0.4 \mathrm{mmol})$ in DMF $(8 \mathrm{~mL})$ was stirred at $100^{\circ} \mathrm{C}$ for 13-15 h. After completion of the reaction, the mixture was poured on to ice water. The product was extracted with ethyl acetate $(2 \times 50 \mathrm{~mL})$. The combined organic phases were washed with water $(2 \times 50 \mathrm{~mL})$ and brine $(50 \mathrm{~mL})$. The organic phase was dried over sodium sulfate and the solvent was evaporated under reduced pressure to give phenylethers (10a-e) in good yields.

4-[2-(2-Fluorophenoxy)ethyl]-2-(4-methoxyphenyl)-5methyl-2,4-dihydro[1,2, 4] triazol-3-one (10a).

Purified by column chromatography (Petroleum ether/ Ethyl acetate, 70/30); white solid; yield: 95\%, m.p. $135-136^{\circ} \mathrm{C}$. ${ }^{1} \mathrm{H}$ NMR (400 MHz, DMSO- $\left.d_{6}\right): \delta 7.74(\mathrm{~d}, 2 \mathrm{H}, J=9.08 \mathrm{~Hz}$, Ar-H), 7.12-7.08 (m, 2H, Ar-H), 7.00-6.94 (m, 4H, Ar-H), $4.17\left(\mathrm{t}, 2 \mathrm{H}, J=5.14 \mathrm{~Hz}, \mathrm{O}-\mathrm{CH}_{2}-\right), 4.02(\mathrm{t}, 2 \mathrm{H}, J=5.14 \mathrm{~Hz}$, triazole-N- $\left.\mathrm{CH}_{2}\right), 3.75\left(\mathrm{~s}, 3 \mathrm{H}, \mathrm{O}-\mathrm{CH}_{3}\right), 2.35\left(\mathrm{~s}, 3 \mathrm{H}, \mathrm{Ar}-\mathrm{CH}_{3}\right)$. MS: $\mathrm{m} / \mathrm{z} 344[\mathrm{~m}+1]$; Anal. Calculated for $\mathrm{C}_{18} \mathrm{H}_{18} \mathrm{FN}_{3} \mathrm{O}_{3}$ : C. 62.97, H. 5.28, N. 12.24. Found C. 62.94, H. 5.29, N. $12.26 \%$.

4-[2-(4-Fluorophenoxy)ethyl]-2-(4-methoxyphenyl)-5methyl-2,4-dihydro[1, 2, 4] triazol-3-one (10b).

Purified by column chromatography (Petroleum ether/ Ethyl acetate, 70/30); white solid; yield: $94 \%, \mathrm{mp} 160-161^{\circ} \mathrm{C}$. ${ }^{1} \mathrm{H}$ NMR (400 MHz, DMSO- $\left.d_{6}\right): \delta 7.75(\mathrm{~d}, 2 \mathrm{H}, J=9.10 \mathrm{~Hz}$, Ar-H), 7.23-7.16 (m, 2H, Ar-H), 7.13-7.08 (m, 1H, Ar-H), $6.98(\mathrm{~d}, 2 \mathrm{H}, J=9.10 \mathrm{~Hz}, \mathrm{Ar}-\mathrm{H}), 6.96-6.93(\mathrm{~m}, 1 \mathrm{H}, \mathrm{Ar}-\mathrm{H})$, $4.25\left(\mathrm{t}, 2 \mathrm{H}, J=5.12 \mathrm{~Hz}, \mathrm{O}-\mathrm{CH}_{2}-\right), 4.07$ (t, $2 \mathrm{H}, J=5.12 \mathrm{~Hz}$, $\mathrm{N}-\mathrm{CH}_{2}-$ ), $3.76\left(\mathrm{~s}, 3 \mathrm{H}, \mathrm{O}-\mathrm{CH}_{3}\right), 2.37$ (s, 3H, triazole- $\left.\mathrm{CH}_{3}\right)$. MS: $\mathrm{m} / \mathrm{z} 343[\mathrm{~m}+1]$; Anal. Calculated for: $\mathrm{C}_{18} \mathrm{H}_{18} \mathrm{FN}_{3} \mathrm{O}_{3}: \mathrm{C}$, 62.97, H, 5.28, N, 12.24, Found: C, 62.98, H, 5.28, N, 12.27 .

4-[2-(Pentafluorophenoxy)ethyl]-2-(4-methoxyphenyl)5-methyl-2,4-dihydro[1, 2, 4] triazol-3-one (10c).

Purified by column chromatography (Petroleum ether/ Ethyl acetate, 65/35); off-white solid; yield: 80\%, m.p. 94-95 ${ }^{\circ} \mathrm{C} .{ }^{1} \mathrm{H}$ NMR (400 MHz, DMSO- $\left.d_{6}\right): \delta 7.73(\mathrm{~d}, 2 \mathrm{H}, J=$ $9.08 \mathrm{~Hz}, \mathrm{Ar}-\mathrm{H}$ ), 7.01 (d, 2H, $=9.08 \mathrm{~Hz}, \mathrm{Ar}-\mathrm{H}$ ), 4.43 (t, 2H, $\left.J=5.04 \mathrm{~Hz}, \mathrm{O}-\mathrm{CH}_{2}-\right), 4.06\left(\mathrm{t}, 2 \mathrm{H}, J=5.04 \mathrm{~Hz}, \mathrm{~N}-\mathrm{CH}_{2}-\right)$, $3.75\left(\mathrm{~s}, 3 \mathrm{H}, \mathrm{O}-\mathrm{CH}_{3}\right), 2.32\left(\mathrm{~s}, 3 \mathrm{H}\right.$, triazole- $\left.\mathrm{CH}_{2}-\right)$. MS: m/z 416 [m+1]; Anal. Calculated for $\mathrm{C}_{18} \mathrm{H}_{14} \mathrm{~F}_{5} \mathrm{~N}_{3} \mathrm{O}_{3}$ : C. 52.06, $\mathrm{H}$. 3.40, N. 10.12 Found: C. 52.10, H. 3.42, N. 10.11\%.

4-[2-(4-Trifluoromethoxyphenoxy)ethyl]-2-(4-methoxyphenyl)-5-methyl-2,4-dihydro[1,2, 4] triazol-3-one (10d).

Purified by column chromatography (Petroleum ether/ Ethyl acetate, 70/30); white solid; yield: $92 \%$, m.p. $152-153^{\circ} \mathrm{C}$. ${ }^{1} \mathrm{H}$ NMR (300 MHz, DMSO- $\left.d_{6}\right): \delta 7.73(\mathrm{~d}, 2 \mathrm{H}, J=9.09 \mathrm{~Hz}$, Ar-H), 7.25-7.04 (m, 4H, Ar-H), 6.98 (d, 2H, $J=9.09 \mathrm{~Hz}$, $\mathrm{Ar}-\mathrm{H}), 4.24\left(\mathrm{t}, 2 \mathrm{H}, \mathrm{J}=5.10 \mathrm{~Hz}, \mathrm{O}-\mathrm{CH}_{2}-\right), 4.06(\mathrm{t}, 2 \mathrm{H}$, $\left.J=5.10 \mathrm{~Hz}, \mathrm{~N}-\mathrm{CH}_{2}-\right), 3.74\left(\mathrm{~s}, 3 \mathrm{H}, \mathrm{O}-\mathrm{CH}_{3}\right), 2.36(\mathrm{~s}, 3 \mathrm{H}$, triazole $\left.-\mathrm{CH}_{3}\right) ;{ }^{13} \mathrm{C}$ NMR $\left(100 \mathrm{MHz}, \mathrm{DMSO}-d_{6}\right): \delta 157.2$,
$156.9,151.7,145.6,142.5,131.5,123.0,121.9,116.2,114.7$, 66.1, 55.7, 41.1, 12.0. MS: m/z 410 [m+1]; Anal. Calculated for $\mathrm{C}_{19} \mathrm{H}_{18} \mathrm{~F}_{3} \mathrm{~N}_{3} \mathrm{O}_{4}$ : C. 55.75, H. 4.43, N. 10.26, Found C. 55.70, H. 4.42 , N. $10.24 \%$.

4-[2-(4-Phenylphenoxy)ethyl]-2-(4-methoxyphenyl)-5methyl-2,4-dihydro[1,2, 4] triazol-3-one (10e).

Purified by column chromatography (Petroleum ether/ Ethyl acetate, 70/30); white solid; yield: $88 \%$, m.p. $133-134^{\circ} \mathrm{C}$. ${ }^{1} \mathrm{H}$ NMR (400 MHz, DMSO- $\left.d_{6}\right): \delta 7.75(\mathrm{~d}, 2 \mathrm{H}, J=9.00 \mathrm{~Hz}$, Ar-H), 7.60-7.58 (m, 4H, Ar-H), 7.44-7.40 (m, 2H, Ar-H), 7.32-7.30 (m, 1H, Ar-H), 7.05-6.99 (m, 4H, Ar-H), $4.26(\mathrm{t}$, $2 \mathrm{H}, J=5.12 \mathrm{~Hz}, \mathrm{O}-\mathrm{CH}_{2}-$ ) , 4.07 (t, $2 \mathrm{H}, J=5.12 \mathrm{~Hz}, \mathrm{~N}-\mathrm{CH}_{2}-$ ), $3.76\left(\mathrm{~s}, 3 \mathrm{H}, \mathrm{O}-\mathrm{CH}_{3}\right), 2.38\left(\mathrm{~s}, 3 \mathrm{H}\right.$, triazole- $\left.\mathrm{CH}_{3}\right) . \mathrm{MS}: \mathrm{m} / \mathrm{z} 402$ [m+1]; Anal. Calculated for $\mathrm{C}_{24} \mathrm{H}_{23} \mathrm{~N}_{3} \mathrm{O}_{3}$ : C. 71.80, H. 5.77, N. 10.47 Found C. 71.91 , H. 10.50, N. 10.45\%.

2.3. General Procedure for the Synthesis of Aniline Derivatives $(11 a-c)$. A mixture of compound (7) $(2 \mathrm{mmol})$, the appropriate substituted anilines $(2.5 \mathrm{mmol})$, and triethylamine ( $3 \mathrm{mmol}$ ) in DMF $(4 \mathrm{~mL})$ was stirred at $115^{\circ} \mathrm{C}$ for $15-16 \mathrm{~h}$. The completion of the reaction was monitored by TLC. The reaction mixture was diluted with water and the product was extracted with ethyl acetate $(2 \times 25 \mathrm{~mL})$. The combined organic phases were washed with water $(2 \times 20 \mathrm{~mL})$ and brine $(20 \mathrm{~mL})$. The organic phase was dried over sodium sulfate and solvent evaporated under reduced pressure to yield the $[1,2,4]$-triazolo aniline derivatives (11a-c) in moderate yields.

4-[2-(4-sec-Butylphenylamino)ethyl]-2-(4-methoxyphenyl)-5-methyl-2,4-dihydro[1,2, 4] triazol-3-one (11a).

Purified by column chromatography (Petroleum ether/ Ethyl acetate, 70/30); off-white solid; yield: 52\%, m.p. 85-86 ${ }^{\circ}$ C. ${ }^{1} \mathrm{H}$ NMR $\left(400 \mathrm{MHz}, \mathrm{DMSO}-d_{6}\right): \delta 7.74(\mathrm{~d}, 2 \mathrm{H}, J=$ $9.14 \mathrm{~Hz}, \mathrm{Ar}-\mathrm{H}), 6.98$ (d, 2H, $J=9.14 \mathrm{~Hz}, \mathrm{Ar}-\mathrm{H}), 6.89$ (d, 2H, $J=8.48 \mathrm{~Hz}, \mathrm{Ar}-\mathrm{H}), 6.53(\mathrm{~d}, 2 \mathrm{H}, J=8.48 \mathrm{~Hz}, \mathrm{Ar}-\mathrm{H}), 5.65(\mathrm{t}$, $1 \mathrm{H}, J=6.28 \mathrm{~Hz}, \mathrm{Ar}-\mathrm{NH}-), 3.75\left(\mathrm{~s}, 3 \mathrm{H},-\mathrm{O}-\mathrm{CH}_{3}\right), 3.75-3.72$ (m, $2 \mathrm{H}$, triazole- $\left.\mathrm{NCH}_{2}-\right) 3.33\left(\mathrm{~m}, 2 \mathrm{H},-\mathrm{NHCH}_{2}-\right), 2.40(\mathrm{~m}$, $1 \mathrm{H}, \mathrm{Ar}-\mathrm{CH}-), 2.15$ (s, 3H, triazole- $\left.\mathrm{CH}_{3}\right), 1.46-1.42(\mathrm{~m}, 2 \mathrm{H}$, $\mathrm{CH}_{3}-\mathrm{CH}_{2}-$ ), 1.22 (d, 3H, $\left.\mathrm{H}=6.96 \mathrm{~Hz}, \mathrm{CH}_{3} \mathrm{CH}-\right), 0.72$ (t, $2 \mathrm{H}, \mathrm{CH}_{3}-\mathrm{CH}_{2}-$ ). MS: m/z 381 [m+1]; Anal. Calculated for $\mathrm{C}_{22} \mathrm{H}_{28} \mathrm{~N}_{4} \mathrm{O}_{2}$ : C. 69.45, H. 7.42, N. 14.72. Found C. 69.40, H. 7.41, N. $14.75 \%$.

4-(4-Methoxyphenyl)-4-[2-(4-methoxyphenylamino)ethyl]-5-methyl-2,4-dihydro[1, 2, 4]triazol-3-one (11b).

Purified by column chromatography (Petroleum ether/ Ethyl acetate, 70/30); brownish liquid; yield: $45 \%,{ }^{1} \mathrm{H}$ NMR $\left(400 \mathrm{MHz}, \mathrm{DMSO}-d_{6}\right): \delta 7.75(\mathrm{~d}, 2 \mathrm{H}, J=9.14 \mathrm{~Hz}, \mathrm{Ar}-\mathrm{H}), 6.99$ $(\mathrm{d}, 2 \mathrm{H}, J=9.14 \mathrm{~Hz}, \mathrm{Ar}-\mathrm{H}), 6.70(\mathrm{~m}, 2 \mathrm{H}, J=8.90 \mathrm{~Hz}, \mathrm{Ar}-\mathrm{H})$, 6.55 (d, 2H, J = 8.90 Hz, Ar-H), 5.46 (t, 1H, Ar-NH-), 3.75 (s, $\left.3 \mathrm{H}, \mathrm{O}-\mathrm{CH}_{3}\right), 3.74-3.72\left(\mathrm{~m}, 2 \mathrm{H}\right.$, triazole- $\left.\mathrm{N}-\mathrm{CH}_{2}-\right), 3.61$ (s, 3H, O- $\mathrm{CH}_{3}$ ), 3.32-3.29 (m, 2H, NH-CH- ) 2.14 (s, 3H, triazole- $\mathrm{CH}_{3}$ ). MS: m/z 355.2 [m+1]; Anal. Calculated for $\mathrm{C}_{19} \mathrm{H}_{22} \mathrm{~N}_{4} \mathrm{O}_{3}$ : C. 64.39, H. 6.26, N. 15.81. Found C. 64.19, H. 6.24, N. $15.80 \%$.

4-[2-(4-Fluorophenylamino)ethyl]-2-(4-methoxyphenyl)-5-methyl-2,4-dihydro[1,2, 4]triazolo-3-one (11c). 
TABLe 1: Antibacterial activity of the compounds $(\mathbf{8}),(\mathbf{9 a}-\mathbf{c}),(\mathbf{1 0 a}-\mathbf{e})$, and $(\mathbf{1 1 a}-\mathbf{c})$.

\begin{tabular}{|c|c|c|c|c|c|}
\hline \multirow{3}{*}{ Compounds } & \multicolumn{3}{|c|}{ MIC of the compounds in $\mu \mathrm{g} / \mathrm{mL}$} & \multirow{2}{*}{\multicolumn{2}{|c|}{ Gram-negative organisms }} \\
\hline & \multicolumn{3}{|c|}{ Gram-positive organisms } & & \\
\hline & B. subtilis & S. aureus & S. epidermidis & E. coli & P. aeruginosa \\
\hline 8 & 18.75 & 18.75 & 18.75 & 18.75 & 18.75 \\
\hline $9 \mathbf{a}$ & 18.75 & 18.75 & 18.75 & 18.75 & 9.75 \\
\hline $9 b$ & 37.5 & 37.5 & 37.5 & 37.5 & 18.75 \\
\hline 9c & 37.5 & 37.5 & 37.5 & 37.5 & 18.75 \\
\hline $10 \mathrm{a}$ & 150 & 150 & 75 & 150 & 75 \\
\hline $10 \mathrm{~b}$ & 150 & 75 & 75 & 150 & 75 \\
\hline $10 \mathrm{c}$ & 150 & 75 & 75 & 150 & 75 \\
\hline 10d & 150 & 75 & 150 & 150 & 37.5 \\
\hline $10 \mathrm{e}$ & 37.5 & 37.5 & 37.5 & 37.5 & 18.75 \\
\hline $11 \mathrm{a}$ & 37.5 & 18.75 & 18.75 & 18.75 & 18.75 \\
\hline $11 b$ & 18.75 & 9.75 & 18.75 & 9.75 & 37.5 \\
\hline $11 \mathrm{c}$ & 150 & 37.5 & 75 & 150 & 37.5 \\
\hline Streptomycin & 6.25 & 1.56 & 1.562 & 3.125 & 3.125 \\
\hline Pencillin & 1.526 & 6.25 & 3.125 & 6.25 & 12.5 \\
\hline
\end{tabular}

TABLe 2: Antifungal activity of the compounds (8), (9a-c), (10a-e), and (11a-c).

\begin{tabular}{|c|c|c|c|c|c|}
\hline \multicolumn{6}{|c|}{ MIC of the compounds in $\mu \mathrm{g} / \mathrm{mL}$} \\
\hline Compound & R. oryzae & A. niger & A. flavus & C. albicans & S. cerevisiae \\
\hline 8 & 18.75 & 18.75 & 150 & 150 & 150 \\
\hline $9 a$ & 37.5 & 150 & 75 & 150 & 150 \\
\hline $9 b$ & 150 & 150 & 150 & 37.5 & 150 \\
\hline $9 c$ & 18.75 & 150 & 150 & 75 & 150 \\
\hline $10 \mathrm{a}$ & 150 & 150 & 150 & 150 & 150 \\
\hline $10 \mathrm{~b}$ & 75 & 75 & 75 & 150 & 150 \\
\hline $10 \mathrm{c}$ & 75 & 37.5 & 75 & 150 & 150 \\
\hline 10d & 37.5 & 37.5 & 150 & 150 & 150 \\
\hline $10 \mathrm{e}$ & 18.75 & 150 & 37.5 & 150 & 150 \\
\hline 11a & 37.5 & 75 & 75 & 150 & 150 \\
\hline $11 b$ & 75 & 37.5 & 75 & 75 & 150 \\
\hline $11 \mathrm{c}$ & 37.5 & 150 & 150 & 150 & 150 \\
\hline Amphotericin-B & 1.562 & 1.56 & 6.25 & 6.25 & 6.25 \\
\hline
\end{tabular}

MIC, $\mu \mathrm{g} / \mathrm{mL}$ : minimum inhibitory concentration.

Purified by column chromatography (Petroleum ether/ Ethyl acetate, 70/30); off-white solid; yield: 50\%, m.p. $88-89^{\circ} \mathrm{C} .{ }^{1} \mathrm{H}$ NMR $\left(400 \mathrm{MHz}, \mathrm{DMSO}-d_{6}\right): \delta 7.75$ (d, $2 \mathrm{H}$, $J=9.14 \mathrm{~Hz}, \mathrm{Ar}-\mathrm{H}), 6.99(\mathrm{~d}, 2 \mathrm{H}, J=9.14 \mathrm{~Hz}, \mathrm{Ar}-\mathrm{H}), 6.91$ $(\mathrm{m}, 2 \mathrm{H}, \mathrm{Ar}-\mathrm{H}), 6.60-6.57(\mathrm{~m}, 2 \mathrm{H}, \mathrm{Ar}-\mathrm{H}), 5.80(\mathrm{t}, 1 \mathrm{H}, J=$ $6.28 \mathrm{~Hz}, \mathrm{Ar}-\mathrm{NH}-), 3.75\left(\mathrm{~s}, 3 \mathrm{H}, \mathrm{O}-\mathrm{CH}_{3}\right), 3.74-3.72(\mathrm{~m}, 2 \mathrm{H}$, triazole- $\left.\mathrm{N}-\mathrm{CH}_{2}-\right)$, 3.32-3.30 (m, $\left.2 \mathrm{H}, \mathrm{NH}-\mathrm{CH}_{2}-\right), 2.14$ (s, $3 \mathrm{H}$, triazole- $\left.\mathrm{CH}_{3}\right)$. MS: $\mathrm{m} / \mathrm{z} 342.8[\mathrm{~m}+1]$; Anal. Calculated for $\mathrm{C}_{18} \mathrm{H}_{19} \mathrm{FN}_{4} \mathrm{O}_{2}$ : C. 63.15, H. 5.59, N. 16.36. Found C. 63.01, H. 5.60 , N. $16.35 \%$.

\section{Biology}

3.1. Antimicrobial Activity. The in vitro antimicrobial activity was carried by using the disc diffusion method [33, 34]. All the newly synthesized compounds were evaluated for their antibacterial activity against Bacillus subtilis, Staphylococcus aureus, Staphylococcus epidermidis, Escherichia coli, and Pseudomonas aeruginosa. The antifungal activity was evaluated against $R$. oryzae, A. niger, A. flavus, C. albicans, and $S$. cerevisiae. The minimum inhibition concentration (MIC) was determined by using the twofold serial dilution method with 64-well microtest plates. The test compounds were dissolved in dimethylformamide (DMF). Further dilutions were made at the required concentrations of 300, 150, 75, $37.5,18.75,9.75,6.25,3.125$, and $1.56 \mu \mathrm{g} / \mathrm{mL}$, respectively. Streptomycin and Penicillin were used as reference standards for antibacterial activity and Amphotericin-B as reference standard for antifungal activity. The standard drugs were selected based on our previous work [1] in order to compare the activity. The results of antibacterial and antifungal activity are given in Tables 1 and 2, respectively. 


\section{Results and Discussion}

From the experimental results, we observed that the $\mathrm{Cs}_{2} \mathrm{CO}_{3}$ works better for compounds (8), (9a-c), and (10a-c) while, for less reactive anilines organic base, triethyl amine works better compared to $\mathrm{Cs}_{2} \mathrm{CO}_{3}$. Hence, triethylamine is used as excellent base for these reactions which in turn increases safety and cost effectiveness.

All the new compounds were characterized by their spectral data. The IR spectra of 1,2,4-triazolo piperidine (8), 1,2,4-triazolo piperazine (9a-c), 1,2,4-triazolo phenylether (10a-e), and 1,2,4-triazolo aniline (11a-c) derivatives show bands in the range $1680-1720 \mathrm{~cm}^{-1}$ corresponding to $\mathrm{C}=\mathrm{O}$ of triazolinone and $1520-1560 \mathrm{~cm}^{-1}$ for $-\mathrm{C}=\mathrm{N}-$. The ${ }^{1} \mathrm{H}$ and ${ }^{13} \mathrm{C}$ NMR spectrum of compound 8 confirmed the conversion from 7 . The $-\mathrm{COCH}_{3}$ proton signals of compound 8 were observed as a singlet at $1.86 \mathrm{ppm}$ and in ${ }^{13} \mathrm{C} \mathrm{NMR}$ the acetyl $\mathrm{C}=\mathrm{O}$ signals were observed at $210 \mathrm{ppm}$. The piperazine proton signals of compounds (9a-c) appeared in the ranges of $3.10-2.90$ and $2.60-2.50 \mathrm{ppm}$. The signals at 6.93 and $7.79 \mathrm{ppm}$ are assigned to the aromatic protons. The compounds $\mathbf{8}$ and $\mathbf{9 a - c}$ gave the stable $\mathrm{M}+1$ ion peaks in mass spectra.

The formation of compounds 10a-e and 11a-c from 7 was confirmed by ${ }^{1} \mathrm{H}$ NMR spectrum. The methylene protons of 10a-e Ar- $\mathrm{OCH}_{2}$ - and triazole-N-CH - were observed in the ranges of 4.45-4.16 and 4.16-4.00 ppm, respectively. The signals in the range 7.84 to $6.74 \mathrm{ppm}$ are due to the aromatic protons. In addition, the two distorted triplets appeared in the ranges 3.80-3.73 and 3.35-3.25 ppm are assigned to the methylene protons. The triplet for single proton of $\mathrm{Ar}-\mathrm{NH}-$ was observed in the range 5.85-5.4 ppm. The peaks in the range 6.78 to $6.50 \mathrm{ppm}$ are assigned to the aromatic protons. The compounds 10a-e and 11a-c were further confirmed by their stable $\mathrm{M}+1$ ion peaks in mass spectra.

From the antibacterial activity results it was observed that compounds $\mathbf{1 1 b}\left(\mathrm{R}_{4}=4-\mathrm{MeO}-\right)$ showed the highest activity against Staphylococcus aureus and Escherichia coli. The compound (9a) $\left(\mathrm{R}_{1}\right.$ and $\left.\mathrm{R}_{2}=\mathrm{Cl}\right)$ showed the highest activity against Pseudomonas aeruginosa. However, relatively lesser activity was noticed in compounds $\mathbf{9 b}$ and $\mathbf{9 c}$ due to the substitution of one or both chlorine atoms in $9 \mathbf{a}$ by fluorine. This difference in activity may be attributed to the presence of bulkier as well as more lipophilic chlorine atoms in 9a. Almost all the newly synthesized compounds have poor activity against all types of fungal strains tested.

\section{Conclusion}

A series of novel [1,2,4]-triazolo piperidine $(\mathbf{8}),[1,2,4]$ triazolo piperazine $(\mathbf{9 a}-\mathbf{c}),[1,2,4]$-triazolo phenylether $(\mathbf{1 0 a}-$ e), and $[1,2,4]$-triazolo aniline (11a-c) derivatives were synthesized in appreciable yields and characterized by NMR, mass spectrometry and IR studies. The compounds 11ac were synthesized using simple and nonhazardous triethylamine base in moderate yields. The newly synthesized compounds were screened for antibacterial and antifungal activity. It was concluded that among all the $[1,2,4]$-triazole derivatives, $\mathbf{1 1 b}(\mathrm{R}=4-\mathrm{MeO}-)$ showed highest activity against Staphylococcus aureus and Escherichia coli. Also, 9a $\left(\mathrm{R}_{1}\right.$ and $\left.\mathrm{R}_{2}=\mathrm{Cl}\right)$ showed the highest activity against Pseudomonas aeruginosa.

\section{References}

[1] B. S. Patil, G. Krishnamurthy, H. B. Naik, P. R. Latthe, and M. Ghate, "Synthesis, characterization and antimicrobial studies of 2-(4-methoxy-phenyl)-5-methyl-4-(2-arylsulfanylethyl)-2,4-dihydro-[1,2,4] triazolo-3-ones and their corresponding sulfones," European Journal of Medicinal Chemistry, vol. 45, no. 8, pp. 3329-3334, 2010.

[2] H. Bayrak, A. Demirbas, N. Demirbas, and S. A. Karaoglu, "Synthesis of some new 1,2,4-triazoles starting from isonicotinic acid hydrazide and evaluation of their antimicrobial activities," European Journal of Medicinal Chemistry, vol. 44, no. 11, pp. 4362-4366, 2009.

[3] V. Padmavathi, P. Thriveni, G. Sudhakar Reddy, and D. Deepti, "Synthesis and antimicrobial activity of novel sulfone-linked bis heterocycles," European Journal of Medicinal Chemistry, vol. 43, no. 5, pp. 917-924, 2008.

[4] B. Soni, M. S. Ranawat, R. Sharma, A. Bhandari, and S. Sharma, "Synthesis and evaluation of some new benzothiazole derivatives as potential antimicrobial agents," European Journal of Medicinal Chemistry, vol. 45, no. 7, pp. 2938-2942, 2010.

[5] J. Xu, Y. Cao, J. Zhang et al., "Design, synthesis and antifungal activities of novel 1,2,4-triazole derivatives," European Journal of Medicinal Chemistry, vol. 46, no. 7, pp. 3142-3148, 2011.

[6] B. Tozkpran, E. Kupeli, E. Yesilada, and M. Ertan, "Preparation of 5-aryl-3-alkylthio-1,2,4-triazoles and corresponding sulfones with antiinflammatory-analgesic activity," Bioorganic and Medicinal Chemistry, vol. 15, no. 4, pp. 1808-1814, 2007.

[7] B. S. Holla, B. Veerandra, M. K. Shivananda, and B. Poojary, "Synthesis characterization and anticancer activity studies on some Mannich bases derived from 1,2,4-triazoles," European Journal of Medicinal Chemistry, vol. 38, no. 7-8, pp. 759-767, 2003.

[8] A. Duran, H. N. Dogan, and S. Rollas, "Synthesis and preliminary anticancer activity of new 1,4-dihydro-3-(3-hydroxy-2naphthyl)-4-substituted-5H-1,2,4-triazoline-5-thiones," Il Farmaco, vol. 57, no. 7, pp. 559-564, 2002.

[9] M. T. Abdel-Aal, W. A. El-Sayed, S. M. El-Kosy, and E. S. H. ElAshry, "Synthesis and antiviral evaluation of novel 5-(N-arylaminomethyl-1,3,4-oxadiazol-2-yl)hydrazines and their sugars, 1,2,4-triazoles, tetrazoles and pyrazolyl derivatives," Archiv der Pharmazie-Chemistry in Life Sciences, vol. 341, no. 5, pp. 307-313, 2008.

[10] I. Kucukguzel, S. G. Kucukguzel, S. Rollas, and M. Kiraz, "Some 3-thioxo/alkylthio-1,2,4-triazoles with a substituted thiourea moiety as possible antimycobacterials," Bioorganic and Medicinal Chemistry Letters, vol. 11, no. 13, pp. 1703-1707, 2001.

[11] A. Foroumadi, Z. Kiani, and F. Soltani, "Antituberculosis agents VIII: synthesis and in vitro antimycobacterial activity of alkyl $\alpha$-[5-(5-nitro-2-thienyl)-1,3,4-thiadiazole-2-ylthio] acetates," Il Farmaco, vol. 58, no. 11, pp. 1073-1076, 2003.

[12] L. Labanauskas, E. Udrenaite, P. Gaidelis, and A. Brukštus, "Synthesis of 5-(2-,3- and 4-methoxyphenyl)-4H-1,2,4triazole-3-thiol derivatives exhibiting anti-inflammatory activity," Il Farmaco, vol. 59, no. 4, pp. 255-259, 2004. 
[13] A. Almasirad, S. A. Tabatabai, M. Faizi et al., "Synthesis and anticonvulsant activity of new 2-substituted-5-[2-(2-fluorophenoxy)phenyl]-1,3,4-oxadiazoles and 1,2,4-triazoles," Bioorganic and Medicinal Chemistry Letters, vol. 14, no. 24, pp. 6057-6059, 2004.

[14] I. Kucukguzel, S. G. Kucukguzel, S. Rollas et al., "Synthesis of some 3-(arylalkylthio)-4-alkyl/aryl-5-(4-aminophenyl)-4H1,2,4-triazole derivatives and their anticonvulsant activity," Il Farmaco, vol. 59, no. 11, pp. 893-901, 2004.

[15] A. Varvaresou, T. Siatra-Papastaikoudi, A. Tsotinis, A. TsantiliKakoulidou, and A. Vamvakides, "Synthesis, lipophilicity and biological evaluation of indole-containing derivatives of 1,3,4thiadiazole and 1,2,4-triazole," Il Farmaco, vol. 53, no. 5, pp. 320-326, 1998.

[16] B. Modzelewska-Banachiewicz, J. Banachiewicz, A. Chodkowska, E. Jagiełło-Wójtowicz, and L. Mazur, "Synthesis and biological activity of new derivatives of 3-(3,4-diaryl-1,2,4triazole-5-yl)propenoic acid," European Journal of Medicinal Chemistry, vol. 39, no. 10, pp. 873-877, 2004.

[17] K. S. S. Lamani, O. Kotresh, M. A. Phaniband, and J. C. Kadakol, "Synthesis, characterization and antimicrobial properties of schiff bases derived from condensation of 8-formyl-7-hydroxy4-methylcoumarin and substituted triazole derivatives," $E$ Journal of Chemistry, vol. 6, no. S1, pp. S239-S246, 2009.

[18] J. Stefanska, M. Stuga, S. Tyski, J. Kossakowski, and M. Dobosz, "Antimicrobial activity of 2,4-dihydro-[1,2,4]triazol3-one derivatives," Polish Journal of Microbiology, vol. 57, no. 2, p. 179, 2008.

[19] M. Shalini, P. Yogeeshwari, D. Sriram, and J. P. Stables, "Cyclization of the semicarbazone template of aryl semicarbazones: synthesis and anticonvulsant activity of 4,5-diphenyl2H-1,2,4-triazol-3(4H)-one," Biomedicine and Pharmacotherapy, vol. 63, no. 3, pp. 187-193, 2009.

[20] J. M. Kane, B. M. Baron, M. W. Dudley, S. M. Sorenson, M. A. Staeger, and F. P. Miller, "2,4-Dihydro-3H-1,2,4-triazol-3-ones as anticonvulsant agents," Journal of Medicinal Chemistry, vol. 33, no. 10, pp. 2772-2777, 1990.

[21] Y. Watanabe, H. Usui, S. Kobayashi et al., "Syntheses and 5HT2 antagonist activity of bicyclic 1,2,4-triazol-3(2H)-one and 1,3,5-triazine-2,4(3H)-dione derivatives," Journal of Medicinal Chemistry, vol. 35, no. 1, pp. 189-194, 1992.

[22] M. Ashok, B. S. Holla, and B. Poojary, "Convenient one pot synthesis and antimicrobial evaluation of some new Mannich bases carrying 4-methylthiobenzyl moiety," European Journal of Medicinal Chemistry, vol. 42, no. 8, pp. 1095-1101, 2007.

[23] A. M. Isloor, B. Kalluraya, and P. Shetty, "Regioselective reaction: synthesis, characterization and pharmacological studies of some new Mannich bases derived from 1,2,4-triazoles," European Journal of Medicinal Chemistry, vol. 44, no. 9, pp. 3784-3787, 2009.

[24] B. S. Holla, K. N. Poojary, and B. S. Rao, "New bis-aminomercaptotriazoles and bis-triazolothiadiazoles as possible anticancer agents," European Journal of Medicinal Chemistry, vol. 37, no. 6, pp. 511-517, 2002.

[25] G. Turan-Zitouni, M. Sivaci, F. S. Kilic, and K. Erol, "Synthesis of some triazolyl-antipyrine derivatives and investigation of analgesic activity," European Journal of Medicinal Chemistry, vol. 36, no. 7-8, pp. 685-689, 2001.

[26] S. Castellano, G. Stefancich, A. Chillotti, and G. Poni, "Synthesis and antimicrobial properties of 3-aryl-1-(1,1' -biphenyl-4-yl)2-(1H-imidazol-1-yl)propanes as 'carba-analogues' of the
$\mathrm{N}$-arylmethyl-N-[(1,1' -biphenyl)-4-ylmethyl])-1H-imidazol1 -amines, a new class of antifungal agents," Il Farmaco, vol. 58, no. 8, pp. 563-568, 2003.

[27] L. Liu and S. Zhu, "A study on the supramolecular structure of inclusion complex of $\beta$-cyclodextrin with prazosin hydrochloride," Carbohydrate Polymers, vol. 68, no. 3, pp. 472-476, 2007.

[28] J. M. Ridley, P. C. Dooley, J. T. Milnes, H. J. Witchel, and J. C. Hancox, "Lidoflazine is a high affinity blocker of the HERG $\mathrm{K}^{+}$channel," Journal of Molecular and Cellular Cardiology, vol. 36, no. 5, pp. 701-705, 2004.

[29] L. Zheng and J. Song, "Voltammetric behavior of urapidil and its determination at multi-wall carbon nanotube paste electrode," Talanta, vol. 73, no. 5, pp. 943-947, 2007.

[30] A. Foroumadi, S. Emami, S. Mansouri et al., "Synthesis and antibacterial activity of levofloxacin derivatives with certain bulky residues on piperazine ring," European Journal of Medicinal Chemistry, vol. 42, no. 7, pp. 985-992, 2007.

[31] A. Foroumadi, S. Ghodsi, S. Emami et al., "Synthesis and antibacterial activity of new fluoroquinolones containing a substituted N-(phenethyl)piperazine moiety," Bioorganic and Medicinal Chemistry Letters, vol. 16, no. 13, pp. 3499-3503, 2006.

[32] P. R. Latthe, V. A. Sunagar, and B. V. Badami, "A simple and efficient synthesis of novel N,N ${ }^{\prime}$-Bis (1H-pyrrol-1-yl)-1-[2(2-aryl-5-methyl-3-oxo-2,4-dihydro-3H-1,2,4-triazol-4-yl) ethyl]-1H-1,2,3-triazole-4,5-dicarboxamides," Journal of Heterocyclic Chemistry, vol. 44, no. 6, pp. 1363-1371, 2007.

[33] R. J. Snow, A. Abeywardane, S. Campbell et al., "Hit-to-lead studies on benzimidazole inhibitors of ITK: discovery of a novel class of kinase inhibitors," Bioorganic and Medicinal Chemistry Letters, vol. 17, no. 13, pp. 3660-3665, 2007.

[34] O. G. Ozden, E. Taner, G. Hakan, and Y. Sulhiye, "Synthesis and antimicrobial activity of some novel phenyl and benzimidazole substituted benzyl ethers," Bioorganic and Medicinal Chemistry Letters, vol. 17, no. 8, pp. 2233-2236, 2007. 

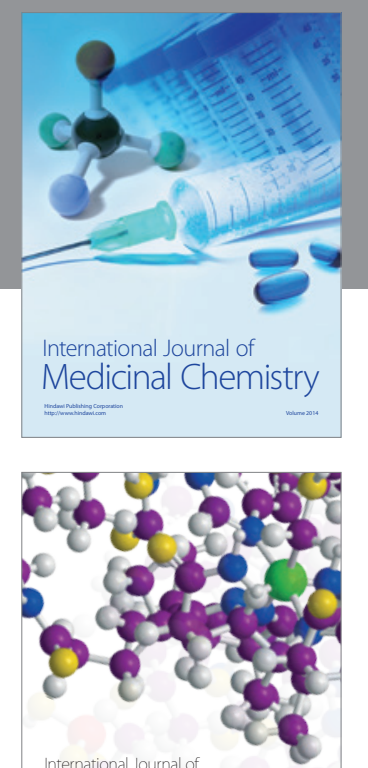

\section{Carbohydrate} Chemistry

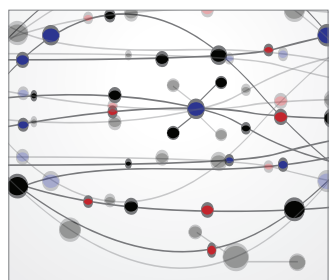

The Scientific World Journal
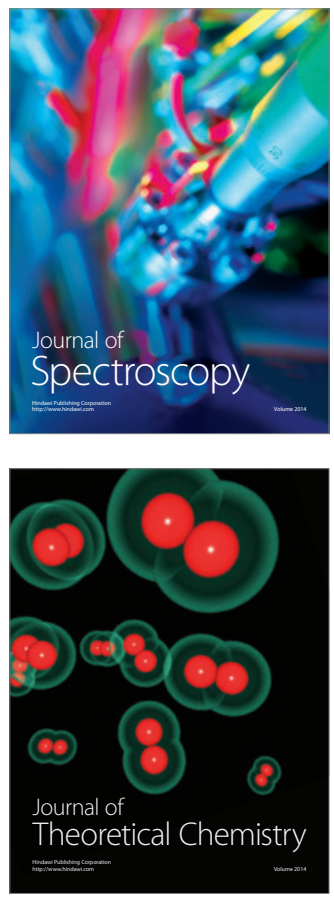
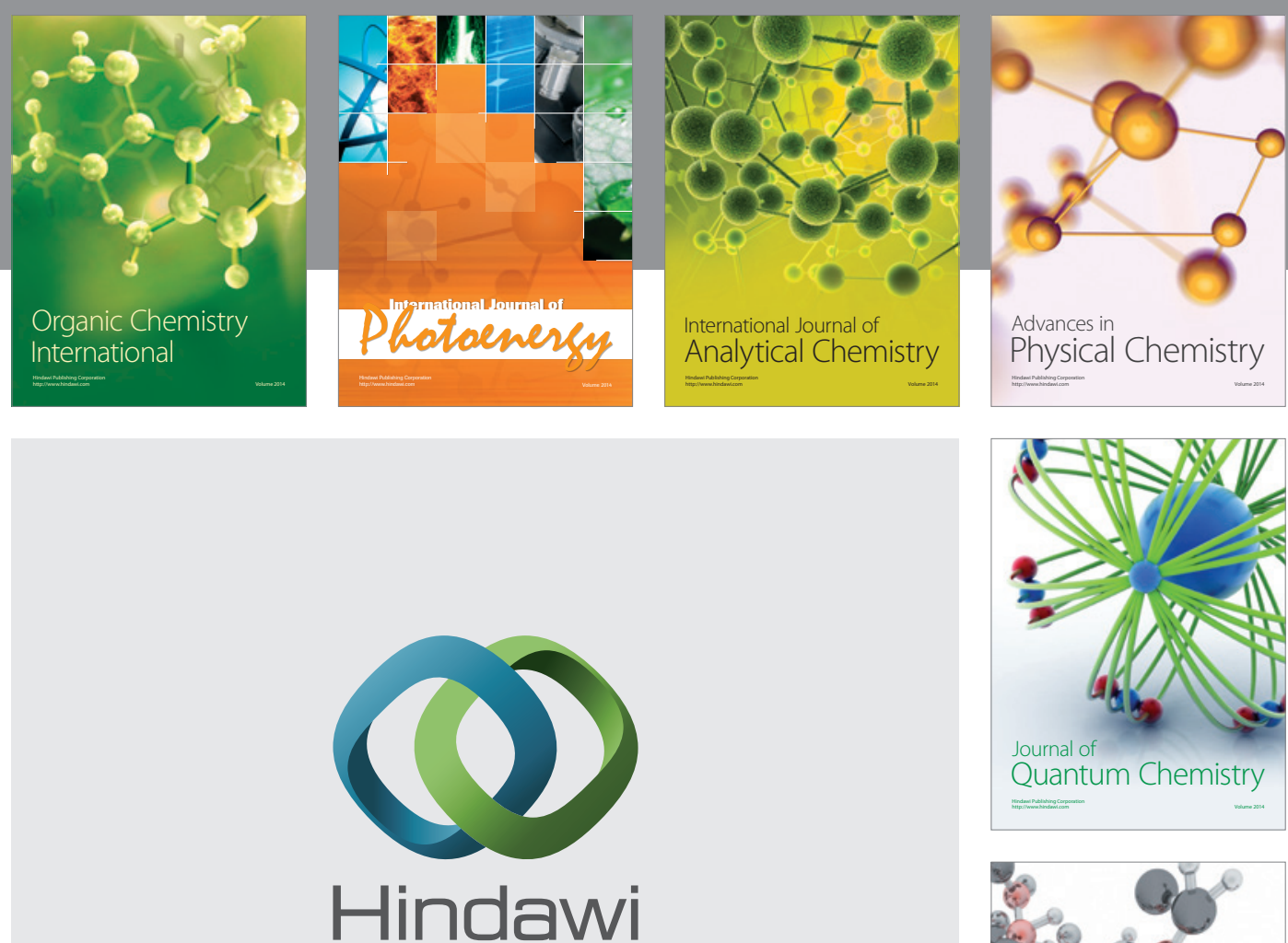

Submit your manuscripts at

http://www.hindawi.com

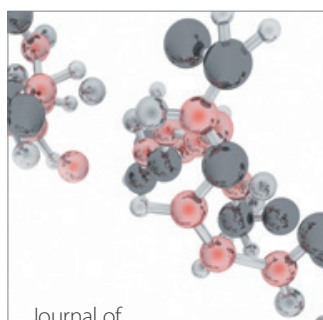

Analytical Methods

in Chemistry

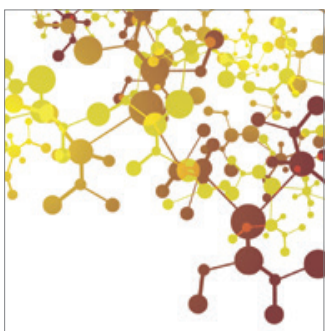

Journal of

Applied Chemistry

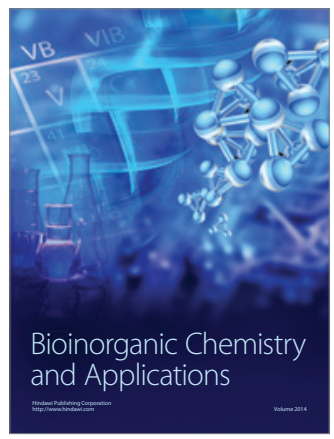

Inorganic Chemistry
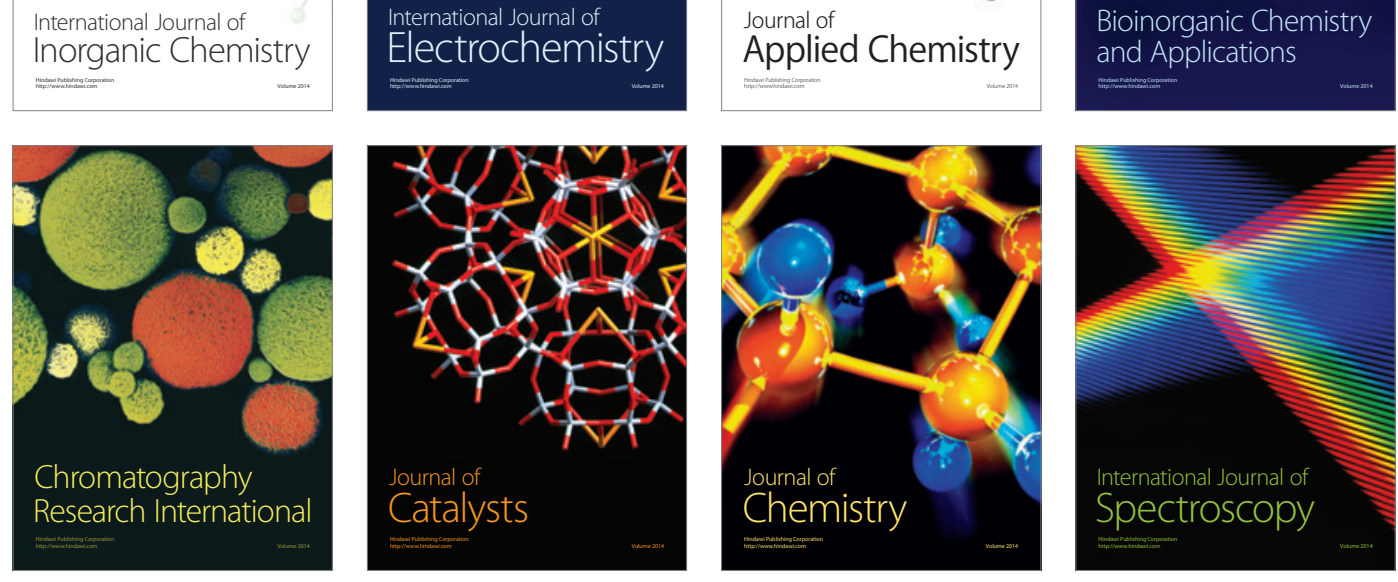